九州大学学術情報リポジトリ

Kyushu University Institutional Repository

\title{
Singular electromagnetic modes in an anisotropic medium
}

Fukumoto, Yasuhide

Faculty of Mathematics and Mathematical Research Center for Industrial Technology, Kyushu University

Samokhin, Alexander B.

Moscow State Institute of Radio Engineering, Electronics and Automatics (MIREA)

http://hdl. handle. net/2324/18935

出版情報 : MI Preprint Series. 2011-1，2011-01-28. 九州大学大学院数理学研究院 バージョン:

権利関係 : 


\title{
MI Preprint Series
}

Kyushu University

The Global COE Program

Math-for-Industry Education \& Research Hub

\section{Singular electromagnetic modes in an anisotropic medium}

\author{
Yasuhide Fukumoto \\ \& Alexander B. Samokhin
}

MI 2011-1

( Received January 28, 2011 )

Faculty of Mathematics

Kyushu University

Fukuoka, JAPAN 


\title{
Singular electromagnetic modes in an anisotropic medium
}

\author{
By Yasuhide Fukumoto ${ }^{1} \dagger$ And Alexander B. Samokhin ${ }^{2}$ \\ ${ }^{1}$ Faculty of Mathematics and Mathematical Research Center for Industrial \\ Technology, Kyushu University, 744 Motooka, Nishi-ku, Fukuoka 819-0395, Japan \\ ${ }^{2}$ Moscow State Institute of Radio Engineering, Electronics and Automatics \\ (MIREA), 78 Vernadsky Avenue, Moscow, 119454, Russia
}

We construct the singular mode corresponding to a spatial essential spectrum of the integral operator for the scattering of the electromagnetic waves by a threedimensional body of finite size with inhomogeneous and anisotropic dielectric permittivity tensor. The permittivity tensor field is assumed to be Hölder continuous throughout the whole space. The singular volume integral equation, transformed from the Maxwell equations, makes it feasible to deduce explicit form of both the continuous essential spectrum and the corresponding singular modes. The obtained singular mode is a natural extension of the previously obtained one for the isotropic case and is applicable to a much wider class of dielectric scattering bodies. A discussion is made of possibility for realizability of the electromagnetic waves, with finite energy, concentrated at a point in the body.

Keywords: Singular integral equation, Continuous spectrum, Singular mode, Electromagnetic wave, Scattering, Plasma

\section{Introduction}

Propagation, refraction and scattering of the electromagnetic waves have been one of key issues in the past several decades during which dramatic evolution of electrocommunication occurred and is even more so in recent years with rapidly developing information technology. The electromagnetic field is ruled by the Maxwell equations. For the scattering phenomena of the electromagnetic waves and possibly related problems as well, it is advantageous to handle, rather than the Maxwell equations, the volume integral equations derived from them from the both aspects of mathematical analyses and numerical computations (Samokhin 2001, 2009).

The mathematical essence of this volume integral operator is encoded in its spatial spectra; their distribution informs us of the existence or non-existence of the solution and of the convergence rate to the solution by an iterative method (Samokhin 2009). Rahola (2000) discovered numerically that eigenvalues of the volume integral operator for the three-dimensional electromagnetic waves scattered by a compact spherical object densely lie on a line, of finite length, for an isotropic medium and on finite lines for an anisotropic medium. Relying on a rigorous mathematical framework developed by Mikhilin (1965) (see also Mikhilin \& Prösdorf 1986), Budko \& Samokhin (2006b) identified this characteristic alignment of eigenvalues as essential and continuous spectra by, above all, writing them down in

$†$ Author for correspondence (yasuhide@math.kyushu-u.ac.jp) 


\title{
MI Preprint Series
}

Kyushu University

The Global COE Program

Math-for-Industry Education \& Research Hub

\section{Singular electromagnetic modes in an anisotropic medium}

\author{
Yasuhide Fukumoto \\ \& Alexander B. Samokhin
}

MI 2011-1

( Received January 28, 2011 )

Faculty of Mathematics

Kyushu University

Fukuoka, JAPAN 
explicit form. In case a monochromatic wave of frequency $\omega(\in \mathbb{R})$ is incident on a dielectric body $Q$ of finite extent, we may introduce the complex dielectric permittivity tensor $\hat{\varepsilon}(\boldsymbol{x})$, represented by a $3 \times 3$ matrix, as functions of the position $\boldsymbol{x}$ inside the scatterer, whose imaginary part, being denoted by $\operatorname{Im}[\cdot]$, has a link with the electro-conductivity tensor $\hat{\sigma}(\boldsymbol{x})$ via $\operatorname{Im}[\hat{\varepsilon}(\boldsymbol{x})]=\hat{\sigma}(\boldsymbol{x}) / \omega$. We take the permittivity of the ambient medium, being looked upon as a vacuum, to be $\varepsilon_{0}$, a scalar, and assume the magnetic permeability to be constant throughout the space including both the scatterer and the ambient medium. With this setting, the essential spectra of the volume integral operator contains the following set of complex numbers $\lambda$ $(\in \mathbb{C})$

$$
\sigma_{\mathrm{s}}=\left\{\lambda=\frac{1}{\varepsilon_{0}} \sum_{m=1}^{3} \sum_{n=1}^{3} \varepsilon_{m n}(\boldsymbol{x}, \omega) q_{m} q_{n} \mid \boldsymbol{x} \in Q, \boldsymbol{q} \in \mathbb{R}^{3} \text { with } q_{1}^{2}+q_{3}^{2}+q_{3}^{2}=1\right\},
$$

in addition to $\lambda=1$. On the assumption of the Hölder continuity of $\hat{\varepsilon}(\boldsymbol{x}, \omega)$ throughout the whole space, the latter $(\lambda=1)$ coincides with the limiting value of $\sigma_{\mathrm{s}}$ at the boundary $\partial Q$ of the scattering body.

For a scatterer comprising isotropic medium, $\varepsilon_{m n}(\boldsymbol{x}, \omega)=\varepsilon(\boldsymbol{x}, \omega) \delta_{m n}$, with $\delta_{m n}$ being Kronecker's delta, (1.1) collapses to the set $\left\{\lambda=\varepsilon(\boldsymbol{x}, \omega) \delta_{m n} / \varepsilon_{0} \mid \boldsymbol{x} \in Q\right\}$. This set generically occupies a finite region in the complex plane, including $\lambda=1$ owing to the Hölder continuity across the boundary $\partial Q$, as its real part $\operatorname{Re}[\lambda]$ and imaginary part $\operatorname{Im}[\lambda]$ are parameterized by three real numbers $\boldsymbol{x}\left(\in \mathbb{R}^{3}\right)$, but when $\varepsilon$ is a constant, it degenerates to a curve connecting $\lambda=1$ and this constant value as demonstrated by Rahola (2000) and Budko \& Samokhin (2006a, 2006b). The isotropic case is special in the sense that, for a give point $\boldsymbol{x}(\in Q)$, the set (1.1) includes only a single element $\lambda=\varepsilon(\boldsymbol{x}, \omega) / \varepsilon_{0}$. For this case, Budko \& Samokhin (2007) constructed, in a tidy form, the singular mode or the singular eigenfunction corresponding to $\lambda=\varepsilon(\boldsymbol{x}, \omega) / \varepsilon_{0}$, which complies with Weyl's definition of the essential spectrum (Hislop \& Sigal 1996). Resemblance of this electromagnetic singular mode with the square root of the Dirac delta function is noteworthy (see also Budko \& Samokhin 2006a).

In a general situation of practical importance, dielectric materials comprise anisotropic media. Media with anisotropic permittivity exhibit by far a richer electromagnetic and optical behaviour as exemplified by plasmas (e.g. Ginzburg 1962) and chiral crystals (e.g. Berry \& Dennis 2003). The modern nano-technology has even manufactured the so called photonic crystals which have negative refraction index (Cubukcu et al. 2003). The goal of the present investigation is to write out in full the singular mode corresponding to the spectra (1.1) for general anisotropic media, by extending the isotropic case (Budko \& Samokhin 2007). This would be one of a few singular modes available explicitly in a tidy form.

We are pursuing the spatial spectra $\lambda$ of the singular integral operator with a given external monochromatic wave of arbitrary real frequency $\omega$, among which the Maxwell equations are restored only when $\lambda=0$. The realizability of $\lambda=0$ as an essential spectrum is a question of our concern to be examined in $\S 5$. Singular modes corresponding to temporal spectra have been addressed in the contexts of waves on and of stability of fluid and plasma motions, but only a few cases admit explicit form of the singular modes. Among them are singular modes of the Vlasov equation for oscillation of a collisionless plasma (Van Kampen 1955; Case 
1959) and of the Rayleigh's equation for waves in the Couette flow (Balmforth and Morrison 1995). Rayleigh's equation is the inviscid limit of the Orr-Sommerfeld equation for the stability of parallel shear flows. In this context, the singular modes have been studied over a century in a different guise of resolving the critical layer and in general do not take simple form (see Maslowe 1986; Balmforth and Morrison 1995; Hirota \& Fukumoto 2008a, 2008b). Van Kampen's mode of the distribution function for plasma oscillation consists of two parts, one of which is a localised function in the velocity space, featured by the Dirac delta function. Our singular mode for the electric field has localised part only. Recently the existence of localised modes, called the trapped modes, has been unveiled for a wide class of waves propagating in a waveguide with obstacles, including water waves (McIver et al. 2001), sound waves (Sugimoto \& Imahori 2006) and electromagnetic waves. The trapped mode corresponds to a point spectrum in isolation or embedded in a continuous spectrum in time (McIver et al. 2001) and is featured by exponential damping in spatial coordinates. In contrast, the singular mode is localised at a point. It cannot be overemphasized that the singular electromagnetic mode satisfies eigen-value equitation for the volume integral operator equivalent to the full Maxwell equations, as opposed to other cases in which a spectrum and the corresponding (singular) mode are the solution of the linearized and thus approximate operator. Notably, if $\lambda=0$ happens to be a spectrum, its (singular) eigenfuction is the solution of the Maxwell equations, implying that the singular mode associated with $\lambda=0$ may have some practical bearing.

In $\S 2$, we give a brief sketch for the deviation of the singular volume integral equation by transforming the Maxwell equations for scattering the electromagnetic waves by a compact body. The singular mode corresponding to the essential spectra (1.1), the main result, is written out in $\S 3$. The proof that this is qualified as the singular mode for the singular volume integral equation follows in $\S 4$. The plasmas are liable to accommodate a diversity of dispersion in the dielectric permittivity tensor. This brings in possibility of containing $\lambda=0$ in the essential spectra (1.1) and thus realizability, in a sense, of the singular mode with the electromagnetic energy localised at a point inside the scattering body. Section 5 gives an illustration for this possibility. The last section $(\S 6)$ is devoted to a summary and conclusions, where we also give a speculation, by a crude mathematical argument, for the mechanism of how concentration of the wave amplitude is brought about.

\section{Singular integral equation and spectra}

We consider scattering of the electromagnetic waves by a body occupying a finite domain $Q$ embedded in the three-dimensional Euclidean space $E^{3}$. The medium constituting the body $Q$ is characterized by a dielectric permittivity $\hat{\varepsilon}$, tensor-valued functions, while the medium outside $Q$ by constant permittivity $\varepsilon_{0}=$ const., a scalar. The magnetic permeability is assumed to be constant everywhere: $\mu=\mu_{0}=$ const. We inquire into the electromagnetic field in the medium $Q$ excited by an external field with time dependence prescribed by factor $\exp (-i \omega t)(\omega \in \mathbb{R})$. The Maxwell equations governing the electric and the magnetic fields $\boldsymbol{E}$ and $\boldsymbol{H}$ are then 
reducible to

$$
\begin{aligned}
& \operatorname{rot} \boldsymbol{E}=\mathrm{i} \omega \mu_{0} \boldsymbol{H}, \quad \operatorname{rot} \boldsymbol{H}=-\mathrm{i} \omega \hat{\varepsilon} \boldsymbol{E}+\boldsymbol{J}^{0}, \\
& \operatorname{div} \hat{\varepsilon} \boldsymbol{E}=0, \quad \operatorname{div} \boldsymbol{H}=0,
\end{aligned}
$$

supplemented by the radiation condition. Here $\boldsymbol{J}^{0}$ is the external current density or the displacement current density, associated with the external field $\boldsymbol{E}^{0}$ and $\boldsymbol{H}^{0}$. We assume that the imaginary part $\operatorname{Im} \varepsilon_{0} \geq 0$ and $\operatorname{Im} \mu_{0} \geq 0$, as it should be.

Introduce the permittivity tensor normalised by the scalar value of the ambient medium:

$$
\hat{\varepsilon}_{\mathrm{r}}(\boldsymbol{x}, \omega)=\hat{\varepsilon}(\boldsymbol{x}, \omega) / \varepsilon_{0} .
$$

To simplify the notation, we write $\hat{\varepsilon}_{\mathrm{r}}(\boldsymbol{x})=\hat{\varepsilon}_{\mathrm{r}}(\boldsymbol{x}, \omega)$ by suppressing the dependence on $\omega$ and make it explicit only when necessary. For both numerical computation and analytical handling, it is sometimes efficient to deal with, rather than the Maxwell equations (2.1) themselves, the volume integro-differential equation for the electric field $\boldsymbol{E}$ in the domain $Q$, derived from (2.1),

$$
\begin{aligned}
\boldsymbol{E}(\boldsymbol{x})-\operatorname{grad} \operatorname{div} \int_{D}\left(\hat{\varepsilon}_{\mathrm{r}}(\boldsymbol{y})-\hat{I}\right) \boldsymbol{E}(\boldsymbol{y}) G(R) \mathrm{d} \boldsymbol{y} \\
-k_{0}^{2} \int_{D}\left(\hat{\varepsilon}_{\mathrm{r}}(\boldsymbol{y})-\hat{I}\right) \boldsymbol{E}(\boldsymbol{y}) G(R) \mathrm{d} \boldsymbol{y}=\boldsymbol{E}^{0}(\boldsymbol{x}) \quad \text { for } \boldsymbol{x} \in Q,
\end{aligned}
$$

where $G$ is the Green function of the Helmholtz equation

$$
G(R)=\frac{\mathrm{e}^{\mathrm{i} k_{0} R}}{4 \pi R}
$$

with $R=|\boldsymbol{x}-\boldsymbol{y}|$ and $k_{0}=\omega \sqrt{\varepsilon_{0} \mu_{0}}$. The efficiency is enhanced by further transforming (2.3) into a singular volume integral equation (Samokhin 2001, 2009):

$$
\begin{aligned}
\boldsymbol{E}(\boldsymbol{x})+\frac{1}{3} & \left(\hat{\varepsilon}_{\mathrm{r}}(\boldsymbol{x})-\hat{I}\right) \boldsymbol{E}(\boldsymbol{x})-\text { p.v. } \int_{Q}\left[\left(\hat{\varepsilon}_{\mathrm{r}}(\boldsymbol{y})-\hat{I}\right) \boldsymbol{E}(\boldsymbol{y}) \cdot \nabla\right] \nabla G(R) \mathrm{d} \boldsymbol{y} \\
& -k_{0}^{2} \int_{Q}\left(\hat{\varepsilon}_{\mathrm{r}}(\boldsymbol{y})-\hat{I}\right) \boldsymbol{E}(\boldsymbol{y}) G(R) \mathrm{d} \boldsymbol{y}=\boldsymbol{E}^{0}(\boldsymbol{x}) \quad \text { for } \boldsymbol{x} \in Q,
\end{aligned}
$$

where the symbol p.v. stands for taking the principal value of the singular integral, that is, a volume integral over the exterior of an infinitesimal ball centered on $\boldsymbol{y}=\boldsymbol{x}$, and $\nabla$ is the gradient operator with respect to $\boldsymbol{x}$. Throughout this paper, we assume that the Cartesian components $\varepsilon_{m n}(\boldsymbol{x})(m, n=1,2,3)$ of the tensor-functions $\hat{\varepsilon}(\boldsymbol{x})$ are Hölder-continuous everywhere, including across the boundary $\partial Q$ of the body, that is,

$$
\left|\varepsilon_{m n}(\boldsymbol{x})-\varepsilon_{m n}(\boldsymbol{y})\right| \leq C|\boldsymbol{x}-\boldsymbol{y}|, \quad\left(\boldsymbol{x}, \boldsymbol{y} \in E^{3}\right)
$$

for some positive constant $C$. For the analysis of the integral equations (2.5), the functional space $L_{2}(Q)$ of square-integrable vector-valued functions with respect to the inner product

$$
(\boldsymbol{u}, \boldsymbol{v})=\int_{Q} \boldsymbol{u}(\boldsymbol{x}) \cdot \boldsymbol{v}^{*}(\boldsymbol{x}) \mathrm{d} \boldsymbol{x},
$$

is the most appropriate, where the asterisk designates the complex conjugate. The following statement is generically true (Samokhin 1990, 2001). 
Theorem 2.1. The operator of the singular integral equation (2.5) is the Fredholm operator in $L_{2}(Q)$ if and only if the following condition

$$
\sum_{m=1}^{3} \sum_{n=1}^{3} \varepsilon_{m n}(\boldsymbol{x}) q_{m} q_{n} \neq 0 \quad(\boldsymbol{x} \in Q)
$$

is satisfied for all combinations of real numbers $q_{1}, q_{2}$ and $q_{3}$ constrained by $|\boldsymbol{q}|^{2}=$ $q_{1}^{2}+q_{3}^{2}+q_{3}^{2}=1$, or the vector $\boldsymbol{q}$ is constrained to the surface $S^{2}$ of the unit sphere.

The spectrum of the operator $\hat{A}$ on the complex plane is the set of such points $\lambda(\in \mathbb{C})$ that the operator $(\hat{A}-\lambda \hat{I})$ does not have an inverse defined everywhere in the Hilbert space $H$. The points $\lambda$ for which the operator $(\hat{A}-\lambda \hat{I})$ is not Fredholm belong to the continuous part of the spectra (the essential spectrum) of $\hat{A}$. The points $\lambda$ such that $(\hat{A}-\lambda \hat{I})$ is a Fredholm operator with the zero index and there exists a nontrivial solution $u$, belong to the discrete part of the spectra of $\hat{A}$.

The integral equation (2.5) may be represented compactly in symbolic form as

$$
\hat{A} \boldsymbol{E}=\boldsymbol{E}-\hat{S}\left(\left(\hat{\varepsilon}_{\mathrm{r}}-\hat{I}\right) \boldsymbol{E}\right)=\boldsymbol{E}^{0} .
$$

For our purpose of searching for the spectra, it is revealing to convert (2.9) into

$$
\hat{A}-\lambda \hat{I}=(1-\lambda)\left[\hat{I}-\hat{S}\left(\frac{\hat{\varepsilon}_{\mathrm{r}}-\lambda \hat{I}}{1-\lambda}-\hat{I}\right)\right] .
$$

Translation of (2.9) and Theorem 1 into the converted form (2.10) tells us that the essential and the continuous part of the spectra of the operator in equation (2.5) contains the values of $\lambda$ satisfying ${ }^{t} \boldsymbol{q}\left(\hat{\varepsilon}_{\mathrm{r}}-\lambda \hat{I}\right) \boldsymbol{q}=0\left(\boldsymbol{q} \in S^{2}\right)$, where the superscript $t$ designates the transpose of the column vector $\boldsymbol{q}$. The collection of these values constitute the set $\sigma_{s}$ defined by (1.1) (Budko \& Samokhin 2006b). Relying upon Mikhilin's approach, Budko \& Samokhin (2006b) showed that $\lambda=1$ also belongs to the essential spectrum. This point $\lambda=1$ is located at the edge of $\sigma_{s}$ since the Hölder continuity (2.6) of the permittivity tensor field $\hat{\varepsilon}(\boldsymbol{x})$ dictates that $\varepsilon_{m n}$ ( $m, n=1,2,3)$ continuously matches, through the boundary of the domain $Q$, to $\delta_{m n} \varepsilon_{0}$, the value of the ambient medium. The set $\sigma_{s}$ is a connected subset in the complex plane.

\section{Electromagnetic singular modes}

In order to find the structure of the modes corresponding to the electromagnetic essential spectrum, we resort here to Weyl's definition of the spectrum (Hislop \& Sigal 1996). This definition states that a number $\lambda(\in \mathbb{C})$ is in the spectrum of the operator $A$ if and only if there exists an infinite sequence $\left\{\psi_{n} \in H\right\}$ in the Hilbert space $H$ such that

$$
\left\|\psi_{n}\right\|=1, \quad \lim _{n \rightarrow \infty}\left\|\hat{A} \psi_{n}-\lambda \psi_{n}\right\|=0 .
$$

Furthermore, $\lambda(\in \mathbb{C})$ is the point of the essential spectrum, if there is a singular sequence satisfying (3.1), that is, a sequence which contains no convergent subsequence. Such a singular sequence does not (strongly) converge to any function from 
$H$, although it may weakly converge to zero. Sequences that do (strongly) converge to some function on $H$ generate eigenfunctions or eigenmodes corresponding to discrete points of the spectra or the eigenvalues. By a formal analogy, we may associate with an essential spectrum, the essential mode as the limit of this singular sequence of functions. An alternative term, which perhaps better reflects the nature and structure of the particular modes to be manipulated subsequently, would be the singular mode or the singular eigenfunction (Budko \& Samokhin 2007).

We take $\boldsymbol{x}=\mathbf{0}$ to be a general position inside $Q$, with no loss of generality, and focus our attention on a point $\lambda(\in \mathbb{C})$ belonging to the essential spectrum (1.1) attained at, some direction $\boldsymbol{q}^{0}=\left(q_{1}^{0}, q_{2}^{0}, q_{3}^{0}\right) \in S^{2}$, the surface of the unit sphere, that is,

$$
\lambda=\frac{1}{\varepsilon_{0}} \varepsilon_{m n}(\mathbf{0}) q_{m}^{0} q_{n}^{0},
$$

where use has been made of Einstein's convention of taking summation from 1 to 3 for repeated indices. In general, the measure of the collection of $\lambda$ may take a finite positive value when the vector $\boldsymbol{q}^{0}$ sweeps out the whole surface of the unit sphere $S^{2}\left(\left|\boldsymbol{q}^{0}\right|=1\right)$, but in cases the trajectory of $\lambda$ draws merely a curve or points; in the isotropic case, the collection of $\lambda$ given by (3.2) shrinks to a point. Alternatively viewed, in a generic case, given a value of $\lambda(\in \mathbb{C})$, the unit vector $\boldsymbol{q}^{0}$ meeting (3.2) constitutes at most a point, because the two dimensional compact surface $S^{2}$ is restricted by the two constraints, the real and the imaginary parts of (3.2).

Consider the operator (2.9), but with the relative dielectric permittivity tensor $\hat{\varepsilon}_{\text {r }}$ replaced by

$$
\hat{\varepsilon}^{\lambda}=\frac{\hat{\varepsilon}_{\mathrm{r}}-\lambda \hat{I}}{1-\lambda},
$$

as suggested by (2.10), and denote it by $\hat{A}^{\lambda}$. Then (3.2) corresponds to

$$
\varepsilon_{m n}^{\lambda}(\mathbf{0}) q_{m}^{0} q_{n}^{0}=0
$$

manifesting the connection between the continuous spectrum (3.2) of $\hat{A}$ and the non-Fredholm property of $\hat{A}^{\lambda}$.

We are now in a position to build the singular mode corresponding to the given continuous spectrum (3.2). For the sake of convenience, we separate the static part from (2.4) as

$$
G(R)=\frac{1}{4 \pi R}+G_{0}(R) ; \quad G_{0}(R)=\frac{\mathrm{e}^{\mathrm{i} k_{0} R}-1}{4 \pi R},
$$

Substituting this expression into (2.5), we deduce a representation of the operator (2.9) and thus of $\hat{A}^{\lambda}$ as

$$
\begin{aligned}
\left(\hat{A}^{\lambda} \boldsymbol{E}\right)(\boldsymbol{x})= & \boldsymbol{E}(\boldsymbol{x})+\frac{1}{3}\left(\hat{\varepsilon}^{\lambda}(\boldsymbol{x})-\hat{I}\right) \boldsymbol{E}(\boldsymbol{x}) \\
& - \text { p.v. } \int_{Q} \frac{\hat{K}(\boldsymbol{x}-\boldsymbol{y})}{R^{3}}\left[\left(\hat{\varepsilon}^{\lambda}(\boldsymbol{y})-\hat{I}\right) \boldsymbol{E}(\boldsymbol{y})\right] \mathrm{d} \boldsymbol{y} \\
& -\int_{Q} \frac{\hat{K}_{0}(\boldsymbol{x}-\boldsymbol{y})}{R^{2}}\left[\left(\hat{\varepsilon}^{\lambda}(\boldsymbol{y})-\hat{I}\right) \boldsymbol{E}(\boldsymbol{y})\right] \mathrm{d} \boldsymbol{y} .
\end{aligned}
$$

Article submitted to Royal Society 
Here the Cartesian components of the tensor fields $\hat{K}(\boldsymbol{x}-\boldsymbol{y})$ and $\hat{K}_{0}(\boldsymbol{x}-\boldsymbol{y})$, being represented by $3 \times 3$ matrices, are defined by

$$
\begin{gathered}
{[\hat{K}(\boldsymbol{x}-\boldsymbol{y})]_{m n}=\frac{1}{4 \pi}\left[\frac{3\left(x_{m}-y_{m}\right)\left(x_{n}-y_{n}\right)}{R^{2}}-\delta_{m n}\right]} \\
{\left[\hat{K}_{0}(\boldsymbol{x}-\boldsymbol{y})\right]_{m n}=\frac{\mathrm{e}^{\mathrm{i} k_{0} R}-1}{4 \pi}\left[\frac{3\left(x_{m}-y_{m}\right)\left(x_{n}-y_{n}\right)}{R^{2}}-\delta_{m n}\right]} \\
+\frac{\mathrm{e}^{\mathrm{i} k_{0} R}}{4 \pi R}\left[-\left(3 i k_{0} R+k_{0}^{2} R^{2}\right) \frac{\left(x_{m}-y_{m}\right)\left(x_{n}-y_{n}\right)}{R^{2}}+\left(k_{0}^{2} R^{2}+i k_{0} R\right) \delta_{m n}\right] .
\end{gathered}
$$

Clearly, $\hat{K}(\boldsymbol{x}-\boldsymbol{y})$ and $\hat{K}_{0}(\boldsymbol{x}-\boldsymbol{y})$ are differentiable tensor-valued functions of the coordinates. It is noteworthy that the kernel $\hat{K}_{0}(\boldsymbol{x}-\boldsymbol{y}) / R^{2}$ produces a compact operator, while that $\hat{K}(\boldsymbol{x}-\boldsymbol{y}) / R^{3}$ produces a singular operator.

The singular mode is expressible in a compact form in the Fourier space. We use the standard form of the Fourier and its inverse transformations

$$
\begin{aligned}
& \tilde{\psi}(\boldsymbol{k})=\mathcal{F}[\psi(\boldsymbol{x})]=(2 \pi)^{-3 / 2} \int_{E^{3}} \psi(\boldsymbol{x}) \mathrm{e}^{-\mathrm{i} \boldsymbol{k} \cdot \boldsymbol{x}} \mathrm{d} \boldsymbol{x}, \\
& \psi(\boldsymbol{x})=\mathcal{F}^{-1}[\tilde{\psi}(\boldsymbol{k})]=(2 \pi)^{-3 / 2} \int_{E^{3}} \tilde{\psi}(\boldsymbol{k}) \mathrm{e}^{\mathrm{i} \boldsymbol{k} \cdot \boldsymbol{x}} \mathrm{d} \boldsymbol{k} .
\end{aligned}
$$

For the given position $\boldsymbol{x}=\mathbf{0}$, we introduce a non-negative function $\varphi(\boldsymbol{q})$ on the unit sphere $\left(\boldsymbol{q} \in S^{2}\right)$ as follows. We extract a small neighborhood, on $S^{2}$, around the point $\boldsymbol{q}^{0}\left(\left|\boldsymbol{q}^{0}\right|=1\right)$ satisfying (3.2), or equivalently satisfying (3.4)

$$
U_{\boldsymbol{q}^{0}}(\alpha)=\left\{\boldsymbol{q} \in S^{2}|| \varepsilon_{l m}^{\lambda}(\mathbf{0}) q_{l} q_{m} \mid \leq \frac{1}{\alpha}\right\},
$$

for some large parameter $\alpha(>0)$ to be specified at the final stage, and denote the solid angle of $U_{\boldsymbol{q}^{0}}(\alpha)$ to be $S(\alpha)$. For an anisotropic medium, $S(\alpha)$ generically decreases in proportion to $\alpha^{-1}$ as $\alpha \rightarrow \infty$. The isotropic case is an exception; $U_{\boldsymbol{q}^{0}}(\alpha)$ covers the whole surface, and hence $S(\alpha)=4 \pi$ independently of $\alpha$, because $\hat{\varepsilon}^{\lambda}(\mathbf{0})$ is $\hat{I}$ multiplied by a scalar.

In order to distinguish, on $S^{2}$, the points contained in $U_{\boldsymbol{q}^{0}}(\alpha)$ from the exterior points, we introduce

$$
\varphi(\boldsymbol{q})=\left\{\begin{array}{cc}
1 & \left(\boldsymbol{q} \in U_{\boldsymbol{q}^{0}}(\alpha)\right) \\
0 & \left(\boldsymbol{q} \notin U_{\boldsymbol{q}^{0}}(\alpha)\right) .
\end{array}\right.
$$

In the Fourier space, the electric field of the singular modes is constructed as a certain limit of

$$
\tilde{\boldsymbol{E}}^{\mathrm{s}}(\boldsymbol{k} ; \alpha, \beta)=\gamma \boldsymbol{k} \exp \left(-\frac{\varphi(\boldsymbol{q})|\boldsymbol{k}|^{2}}{\alpha}-\frac{(1-\varphi(\boldsymbol{q}))|\boldsymbol{k}|^{2}}{\beta}\right),
$$

where $\boldsymbol{q}=\boldsymbol{k} /|\boldsymbol{k}|$, and $\alpha, \beta$ and $\gamma$ are constants to be prescribed below. The representation of the electric field in the real space $E^{3}$ is then obtained as the limit of

$$
\boldsymbol{E}^{\mathrm{s}}(\boldsymbol{x} ; \alpha, \beta)=\mathcal{F}^{-1}\left[\tilde{\boldsymbol{E}}^{\mathrm{s}}(\boldsymbol{k} ; \alpha, \beta)\right] .
$$


The coefficient $\gamma(\in \mathbb{R})$ is determined so as for the electric field to comply with the normalisation condition

$$
\|\boldsymbol{E}(\boldsymbol{x})\|_{\bar{L}^{2}\left(E^{3}\right)}=1,
$$

resulting in

$$
\gamma^{2} \frac{3 \sqrt{\pi}}{32 \sqrt{2}}\left\{S(\alpha) \alpha^{5 / 2}+[4 \pi-S(\alpha)] \beta^{5 / 2}\right\}=1,
$$

which is to be shown later as (4.2). For the isotropic case, $\varphi(\boldsymbol{q}) \equiv 1$ identically and $S(\alpha) \equiv 4 \pi$, whence (3.11) and (3.12), with $\gamma$ provided by (3.14), are reduced to the representations of the singular modes obtained by Budko \& Samokhin (2007).

In the next section, we shall show that, by taking the limit of $\beta \rightarrow \infty$, with a constraint

$$
\alpha=\beta^{\tau}(\tau>5 / 3)
$$

being reflective of $S(\alpha)=O\left(\alpha^{-1}\right)$ as $\alpha \rightarrow \infty$, (3.11) gives rise to the Fourier representation of the singular mode. The electric field $\boldsymbol{E}^{\mathrm{s}}(\boldsymbol{x})$ of the singular mode in the real space $E^{3}$ is obtained from its inverse transform (3.12). Clearly, the amplitude of the singular mode, obtained when parameters $\alpha$ and $\beta$ tend to infinity. is localised at the point $\boldsymbol{x}=\mathbf{0}$, though the total electric energy is finite as dictated by (3.13).

\section{Proof of singular modes}

In the following, the limiting sequence of (3.12) as the parameters $\alpha$ and $\beta$ are varied is shown to meet the definition (3.1) of the singular mode in Weyl's sense. Along the same line of the procedure with the isotropic case (Budko \& Samokhin 2007), we can verify that the sequence $\left\{\boldsymbol{E}^{\mathrm{s}}\left(\boldsymbol{x} ; \alpha_{n}, \beta_{n}\right)\right\}$ does not have a convergent subsequence in the Hilbert space when $\alpha_{n}$ and $\beta_{n}(n \in \mathbb{N})$ are monotonically increased without limit in the way as prescribed by (3.15):

$$
\alpha_{n}=\beta_{n}^{\tau}(\tau>5 / 3), \quad \lim _{n \rightarrow \infty} \beta_{n}=+\infty, \beta_{n+1}>\beta_{n},
$$

As $\alpha$ and $\beta$ are increased, $\boldsymbol{E}^{\mathrm{s}}(\boldsymbol{x})$ defined by (3.12) tends to be localised to a narrower region around the point $\boldsymbol{x}=\mathbf{0}$. Consequently, we have

$$
\begin{aligned}
& \left\|\boldsymbol{E}^{\mathrm{s}}(\boldsymbol{x})\right\|_{L^{2}(Q)}^{2} \approx\left\|\boldsymbol{E}^{\mathrm{s}}(\boldsymbol{x})\right\|_{L^{2}\left(E^{3}\right)}^{2}=\left\|\tilde{\boldsymbol{E}}^{\mathrm{s}}(\boldsymbol{k})\right\|_{L^{2}\left(E^{3}\right)}^{2}=\int_{S^{2}} \int_{0}^{\infty} k^{2}\left|\tilde{\boldsymbol{E}}^{\mathrm{s}}(\boldsymbol{k})\right| \mathrm{d} k \mathrm{~d} \Omega \\
& =\gamma^{2} S(\alpha) \int_{0}^{\infty} k^{4} \exp \left(-\frac{2 k^{2}}{\alpha}\right) \mathrm{d} k+\gamma^{2}(4 \pi-S(\alpha)) \int_{0}^{\infty} k^{4} \exp \left(-\frac{2 k^{2}}{\beta}\right) \mathrm{d} k,
\end{aligned}
$$

upon substitution from (3.11) for evaluation of the integral $\int_{S^{2}} \mathrm{~d} \Omega$ over the surface of the unit sphere. The first equality $(\approx)$ becomes exact in the limit of $\alpha, \beta \rightarrow \infty$, since the electric field is completely confined in $Q$. Using

$$
\int_{0}^{\infty} k^{4} \exp \left(-\rho k^{2}\right) \mathrm{d} k=\frac{3 \sqrt{\pi}}{8 \rho^{5 / 2}},
$$

Article submitted to Royal Society 
for an arbitrary positive constant $\rho$, the enforcement of the normalisation condition (3.13) yields (3.14). We read off, from (2.10), $\hat{A}-\lambda \hat{I}=(1-\lambda) \hat{A}^{\lambda}$. As $\lambda=1$ is regarded as an exceptional value, all we have to do is to show

$$
\lim _{n \rightarrow \infty}\left\|\hat{A}^{\lambda} \boldsymbol{E}^{\mathrm{S}}\left(\boldsymbol{x} ; \alpha_{n}, \beta_{n}\right)\right\|_{L^{2}(Q)}=0
$$

in the constrained limit (4.1), with the unit vector $\boldsymbol{q}^{0}$ specified by (3.2).

For this purpose, we rearrange (3.6) as

$$
\begin{aligned}
\left(\hat{A}^{\lambda} \boldsymbol{E}^{\mathrm{S}}\right)(\boldsymbol{x})= & \boldsymbol{E}^{\mathrm{S}}(\boldsymbol{x})+\frac{1}{3}\left(\hat{\varepsilon}^{\lambda}(\mathbf{0})-\hat{I}\right) \boldsymbol{E}^{\mathrm{S}}(\boldsymbol{x}) \\
- \text { p.v. } & \int_{Q} \frac{\hat{K}(\boldsymbol{x}-\boldsymbol{y})}{R^{3}}\left[\left(\hat{\varepsilon}^{\lambda}(\mathbf{0})-\hat{I}\right) \boldsymbol{E}^{\mathrm{S}}(\boldsymbol{y})\right] \mathrm{d} \boldsymbol{y}+\frac{1}{3}\left(\hat{\varepsilon}^{\lambda}(\boldsymbol{x})-\hat{\varepsilon}^{\lambda}(\mathbf{0})\right) \boldsymbol{E}^{\mathrm{S}}(\boldsymbol{x}) \\
& - \text { p.v. } \int_{Q} \frac{\hat{K}(\boldsymbol{x}-\boldsymbol{y})}{R^{3}}\left[\left(\hat{\varepsilon}^{\lambda}(\boldsymbol{y})-\hat{\varepsilon}^{\lambda}(\mathbf{0})\right) \boldsymbol{E}^{\mathrm{S}}(\boldsymbol{y})\right] \mathrm{d} \boldsymbol{y} \\
& -\int_{Q} \frac{\hat{K}_{0}(\boldsymbol{x}-\boldsymbol{y})}{R^{2}}\left[\left(\hat{\varepsilon}^{\lambda}(\boldsymbol{y})-\hat{I}\right) \boldsymbol{E}^{\mathrm{S}}(\boldsymbol{y})\right] \mathrm{d} \boldsymbol{y} .
\end{aligned}
$$

It follows immediately from (4.5) that

$$
\left\|\hat{A}^{\lambda} \boldsymbol{E}^{\mathrm{S}}\right\|_{L^{2}(Q)} \leq\left\|\boldsymbol{F}_{1}\right\|_{L^{2}(Q)}+\left\|\boldsymbol{F}_{2}\right\|_{L^{2}(Q)}+\left\|\boldsymbol{F}_{3}\right\|_{L^{2}(Q)}+\left\|\boldsymbol{F}_{4}\right\|_{L^{2}(Q)},
$$

where, for $\boldsymbol{x} \in Q$,

$$
\begin{aligned}
\boldsymbol{F}_{1}(\boldsymbol{x})= & \boldsymbol{E}^{\mathrm{s}}(\boldsymbol{x})+\frac{1}{3}\left(\hat{\varepsilon}^{\lambda}(\mathbf{0})-\hat{I}\right) \boldsymbol{E}^{\mathrm{s}}(\boldsymbol{x}) \\
& - \text { p.v. } \int_{Q} \frac{\hat{K}(\boldsymbol{x}-\boldsymbol{y})}{R^{3}}\left[\left(\hat{\varepsilon}^{\lambda}(\mathbf{0})-\hat{I}\right) \boldsymbol{E}^{\mathrm{s}}(\boldsymbol{y})\right] \mathrm{d} \boldsymbol{y}, \\
\boldsymbol{F}_{2}(\boldsymbol{x})= & \frac{1}{3}\left(\hat{\varepsilon}^{\lambda}(\boldsymbol{x})-\hat{\varepsilon}^{\lambda}(\mathbf{0})\right) \boldsymbol{E}^{\mathrm{s}}(\boldsymbol{x}), \\
\boldsymbol{F}_{3}(\boldsymbol{x})= & - \text { p.v. } \int_{Q} \frac{\hat{K}(\boldsymbol{x}-\boldsymbol{y})}{R^{3}}\left[\left(\hat{\varepsilon}^{\lambda}(\boldsymbol{y})-\hat{\varepsilon}^{\lambda}(\mathbf{0})\right) \boldsymbol{E}^{\mathrm{s}}(\boldsymbol{y})\right] \mathrm{d} \boldsymbol{y}, \\
\boldsymbol{F}_{4}(\boldsymbol{x})= & -\int_{Q} \frac{\hat{K}_{0}(\boldsymbol{x}-\boldsymbol{y})}{R^{2}}\left[\left(\hat{\varepsilon}^{\lambda}(\boldsymbol{y})-\hat{I}\right) \boldsymbol{E}^{\mathrm{s}}(\boldsymbol{y})\right] \mathrm{d} \boldsymbol{y} .
\end{aligned}
$$

Comparison of (2.3) with (2.5) shows that

$$
\begin{gathered}
\frac{1}{3}\left(\hat{\varepsilon}^{\lambda}(\mathbf{0})-\hat{I}\right) \boldsymbol{E}^{\mathrm{S}}(\boldsymbol{x})-\text { p.v. } \int_{D} \frac{\hat{K}(\boldsymbol{x}-\boldsymbol{y})}{R^{3}}\left[\left(\hat{\varepsilon}^{\lambda}(\mathbf{0})-\hat{I}\right) \boldsymbol{E}^{\mathrm{S}}(\boldsymbol{y})\right] \mathrm{d} \boldsymbol{y} \\
=-\operatorname{grad} \operatorname{div} \int_{D}\left(\hat{\varepsilon}^{\lambda}(\mathbf{0})-\hat{I}\right) \boldsymbol{E}^{\mathrm{S}}(\boldsymbol{y}) \frac{1}{4 \pi R} \mathrm{~d} \boldsymbol{y},
\end{gathered}
$$

for arbitrary domain $D$ in $E^{3}$. With the help of (4.11), the first term (4.7) is decomposed into

$$
\boldsymbol{F}_{1}(\boldsymbol{x})=\boldsymbol{F}_{11}(\boldsymbol{x})+\boldsymbol{F}_{12}(\boldsymbol{x}) \text { for } \boldsymbol{x} \in Q
$$


where

$$
\begin{gathered}
\boldsymbol{F}_{11}(\boldsymbol{x})=\boldsymbol{E}^{\mathrm{s}}(\boldsymbol{x})-\operatorname{grad} \operatorname{div} \int_{E^{3}}\left(\hat{\varepsilon}^{\lambda}(\mathbf{0})-\hat{I}\right) \boldsymbol{E}^{\mathrm{s}}(\boldsymbol{y}) \frac{1}{4 \pi R} \mathrm{~d} \boldsymbol{y}, \\
\boldsymbol{F}_{12}(\boldsymbol{x})=\text { p.v. } \int_{E^{3}} m(\boldsymbol{y}) \frac{\hat{K}(\boldsymbol{x}-\boldsymbol{y})}{R^{3}}\left[\left(\hat{\varepsilon}^{\lambda}(\mathbf{0})-\hat{I}\right) \boldsymbol{E}^{\mathrm{s}}(\boldsymbol{y})\right] \mathrm{d} \boldsymbol{y} ; \\
m(\boldsymbol{y})= \begin{cases}0 & \text { for } \boldsymbol{y} \in Q \\
1 & \text { for } \boldsymbol{y} \notin Q .\end{cases}
\end{gathered}
$$

Since the Fourier transform (3.8) maintains the norm, $\left\|\boldsymbol{F}_{11}\right\|_{L^{2}\left(E^{3}\right)}=\left\|\tilde{\boldsymbol{F}}_{11}\right\|_{L^{2}\left(E^{3}\right)}$, and therefore $\left\|\boldsymbol{F}_{1}\right\|_{L^{2}(Q)} \leq\left\|\tilde{\boldsymbol{F}}_{11}\right\|_{L^{2}\left(E^{3}\right)}+\left\|\boldsymbol{F}_{12}\right\|_{L^{2}\left(E^{3}\right)}$. Each term is shown to vanish in the limit, with the constraint (3.15), as follows.

From the fact that the function

$$
V_{0}(\boldsymbol{x})=\int_{E^{3}} \frac{1}{4 \pi R} U(\boldsymbol{y}) \mathrm{d} \boldsymbol{y}
$$

satisfies the Poisson equation $\Delta V_{0}=-U$, we manipulate identities

$$
\begin{aligned}
& \mathcal{F}\left[\int_{E^{3}} \frac{1}{4 \pi R} U(\boldsymbol{y}) \mathrm{d} \boldsymbol{y}\right]=\frac{\mathcal{F}[U]}{|\boldsymbol{k}|^{2}}, \\
& \mathcal{F}\left[\frac{\partial}{\partial x_{m}} \frac{\partial}{\partial x_{n}} \int_{E^{3}} \frac{1}{4 \pi R} U(\boldsymbol{y}) \mathrm{d} \boldsymbol{y}\right]=-q_{m} q_{n} \mathcal{F}[U],
\end{aligned}
$$

by use of the definition $q_{m}=k_{m} /|\boldsymbol{k}|$. Upon substitution from the Fourier representation (3.11), the Fourier transform of the $n$-th component $F_{11 n}(\boldsymbol{x})$ of (4.13) simplifies, by virtue of (4.16) and $q_{l} q_{l}=1$, to

$$
\mathcal{F}\left[F_{11 n}\right]=\left(\delta_{n l}+q_{n} q_{m} \varepsilon_{m l}^{\lambda}(\mathbf{0})-q_{n} q_{l}\right) \mathcal{F}\left[E_{l}^{\mathrm{s}}\right]=\left(\varepsilon_{m l}^{\lambda}(\mathbf{0}) q_{m} q_{l}\right) \tilde{E}_{n}^{\mathrm{s}}(\boldsymbol{k}) .
$$

We employ, as the norm of a tensor, the standard definition

$$
\left\|\hat{\varepsilon}^{\lambda}(\mathbf{0})\right\|=\left(\sum_{l=1}^{3} \sum_{m=1}^{3}\left|\varepsilon_{m l}^{\lambda}(\mathbf{0})\right|^{2}\right)^{1 / 2} \text {. }
$$

By taking the advantage of (4.17) with $\tilde{E}_{n}^{\mathrm{s}}(\boldsymbol{k})$ substituted from (3.11), the norm of the Fourier representation $\tilde{\boldsymbol{F}}_{11}$ and thus that of $\boldsymbol{F}_{11}$ itself are bounded above as

$$
\begin{aligned}
\left\|\tilde{\boldsymbol{F}}_{11}(\boldsymbol{k})\right\|_{L^{2}\left(E^{3}\right)}^{2} \leq & \frac{\gamma^{2}}{\alpha^{2}} S(\alpha) \int_{0}^{\infty} k^{4} \exp \left(-\frac{2 k^{2}}{\alpha}\right) \mathrm{d} k \\
& +\gamma^{2}\left\|\hat{\varepsilon}^{\lambda}(\mathbf{0})\right\|^{2}(4 \pi-S(\alpha)) \int_{0}^{\infty} k^{4} \exp \left(-\frac{2 k^{2}}{\beta}\right) \mathrm{d} k \\
= & \gamma^{2} \frac{3 \sqrt{\pi}}{32 \sqrt{2}}\left[S(\alpha) \sqrt{\alpha}+\left\|\hat{\varepsilon}^{\lambda}(\mathbf{0})\right\|^{2}(4 \pi-S(\alpha)) \beta^{5 / 2}\right] .
\end{aligned}
$$

Recalling the definition of (3.14) of $\gamma$ and that $S(\alpha)=O\left(\alpha^{-1}\right)$ as $\alpha \rightarrow \infty$, $\left\|\tilde{\boldsymbol{F}}_{11}(\boldsymbol{k})\right\|_{L^{2}\left(E^{3}\right)}^{2} \rightarrow 0$ in the constrained limit (3.15), $\alpha=\beta^{2}$ say. This completes the major part of the proof. In passing, we give a remark on the isotropic case. From 
the definition (3.9), $S(\alpha)=4 \pi$, for which only the first term survives in (4.19), and (3.14) obtained by the normalisation condition (3.13) informs us of $\gamma^{2}$ being proportional to $S(\alpha)^{-1} \alpha^{-5 / 2}$. Accordingly, the right-hand side (4.19) tends to zero in the limit of $\alpha \rightarrow \infty$. There is a subtle case between the anisotropic case treated above and the isotropic case, namely the case where the components $\varepsilon_{m n}^{\lambda}(\mathbf{0})$ are infinitely small or so small that $S(\alpha)=O\left(\alpha^{-a}\right)(0<a<1)$ as $\alpha \rightarrow \infty$. As is evident, the conclusion remains unchanged in this intermediate case.

The estimation of the remaining term $\boldsymbol{F}_{12}$ of (4.12) is straightforward.

$$
\begin{gathered}
\left\|\boldsymbol{F}_{12}(\boldsymbol{x})\right\|_{L^{2}\left(E^{3}\right)}^{2} \leq\left\|\hat{K}_{\mathrm{s}}\right\|^{2} \int_{E^{3} \backslash Q}\left|\left(\hat{\varepsilon}^{\lambda}(\mathbf{0})-\hat{I}\right) \boldsymbol{E}^{\mathrm{s}}(\boldsymbol{x} ; \alpha, \beta)\right|^{2} \mathrm{~d} \boldsymbol{x} \\
\leq\left\|\hat{K}_{\mathrm{s}}\right\|^{2}\left\|\hat{\varepsilon}^{\lambda}(\mathbf{0})-\hat{I}\right\|^{2} \int_{E^{3} \backslash Q}\left|\boldsymbol{E}^{\mathrm{s}}(\boldsymbol{x} ; \alpha, \beta)\right|^{2} \mathrm{~d} \boldsymbol{x} .
\end{gathered}
$$

Here $\left\|\hat{K}_{\mathrm{s}}\right\|$ is the norm of the kernel $\hat{K}(\boldsymbol{x}-\boldsymbol{y}) / R^{3}$ of the singular integral operator in the functional space $L^{2}\left(E^{3}\right)$, appearing in (3.6). This value is shown to be a bounded (Samokhin 2001). Hence the right-hand side of (4.20) decreases to 0 as $\alpha, \beta \rightarrow \infty$, since $\boldsymbol{E}^{\mathrm{s}}(\boldsymbol{x} ; \alpha, \beta)$ is confined in $Q$ around the singular point $\boldsymbol{x}=\mathbf{0}$ in this limit.

Next we turn to $\boldsymbol{F}_{2}(\boldsymbol{x})$ defined by (4.8). The localisation of (3.12), the inverse Fourier transform of (3.11), for large values of $\alpha$ and $\beta$ is mathematically featured as follows. We denote $\Omega_{\delta}$ to be a ball of radius $\delta$ centered on the origin $\boldsymbol{x}=\mathbf{0}$. For $\forall \beta(>0), \exists \varepsilon(\delta, \beta), \exists B(\beta)$, some positive decreasing of $\beta$ and $\exists \alpha=\alpha(\beta)$, some positive increasing function of $\beta$, such that, if $\delta>B(\beta)$,

$$
\left\|\boldsymbol{E}^{\mathrm{S}}(\boldsymbol{x} ; \alpha, \beta)\right\|_{L^{2}\left(E^{3} \backslash \Omega_{\delta}\right)} \leq \varepsilon(\delta, \beta) .
$$

where $\lim _{\beta \rightarrow \infty} B(\beta)=0, \lim _{\beta \rightarrow \infty} \alpha(\beta)=\infty$ and $\lim _{\beta \rightarrow \infty} \varepsilon(\delta, \beta)=0$. Moreover, the limit of $\beta \rightarrow \infty$ allows us to take the limit of $\delta \rightarrow 0$. Taking account of (4.21) and the assumption (2.6) indicating the Hölder continuity of $\hat{\varepsilon}^{\lambda}(\boldsymbol{x})$ over the whole space, we obtain

$$
\begin{aligned}
\left\|\boldsymbol{F}_{2}(\boldsymbol{x})\right\|_{L^{2}(Q)}^{2} \leq\left\|\boldsymbol{F}_{2}(\boldsymbol{x})\right\|_{L^{2}\left(E^{3}\right)}^{2}=\frac{1}{9} \int_{\Omega_{\delta}}\left|\left(\hat{\varepsilon}^{\lambda}(\boldsymbol{x})-\hat{\varepsilon}^{\lambda}(\mathbf{0})\right) \boldsymbol{E}^{\mathrm{s}}(\boldsymbol{x} ; \alpha, \beta)\right|^{2} \mathrm{~d} \boldsymbol{x} \\
\quad+\frac{1}{9} \int_{E^{3} \backslash \Omega_{\delta}}\left|\left(\hat{\varepsilon}^{\lambda}(\boldsymbol{x})-\hat{\varepsilon}^{\lambda}(\mathbf{0})\right) \boldsymbol{E}^{\mathrm{S}}(\boldsymbol{x} ; \alpha, \beta)\right|^{2} \mathrm{~d} \boldsymbol{x} \\
\leq \quad \frac{1}{9}\left(\max _{\boldsymbol{x} \in \Omega_{\delta}}\left\|\hat{\varepsilon}^{\lambda}(\boldsymbol{x})-\hat{\varepsilon}^{\lambda}(\mathbf{0})\right\|^{2} \int_{\Omega_{\delta}}\left|\boldsymbol{E}^{\mathrm{S}}(\boldsymbol{x} ; \alpha, \beta)\right|^{2} \mathrm{~d} \boldsymbol{x}\right. \\
\left.\quad+\max _{\boldsymbol{x} \in E^{3} \backslash \Omega_{\delta}}\left\|\hat{\varepsilon}^{\lambda}(\boldsymbol{x})-\hat{\varepsilon}^{\lambda}(\mathbf{0})\right\|^{2} \int_{E^{3} \backslash \Omega_{\delta}}\left|\boldsymbol{E}^{\mathrm{S}}(\boldsymbol{x} ; \alpha, \beta)\right|^{2} \mathrm{~d} \boldsymbol{x}\right) \\
\leq \quad C \delta^{2} \int_{\Omega_{\delta}}\left|\boldsymbol{E}^{\mathrm{S}}(\boldsymbol{x} ; \alpha, \beta)\right|^{2} \mathrm{~d} \boldsymbol{x}+\max _{\boldsymbol{x} \in E^{3} \backslash \Omega_{\delta}}\left\|\hat{\varepsilon}^{\lambda}(\boldsymbol{x})-\hat{\varepsilon}^{\lambda}(\mathbf{0})\right\|^{2} \varepsilon(\delta, \beta) .
\end{aligned}
$$

The last expression approaches 0 by taking the limit of $\delta \rightarrow 0$, in keeping with the constrained limit of $\alpha, \beta \rightarrow \infty$, and by taking the finiteness (3.13) of the norm of the electric field into consideration. Comparing (4.8) and (4.9), we are led to

$$
\left\|\boldsymbol{F}_{3}(\boldsymbol{x})\right\|_{L^{2}(Q)}^{2} \leq\left\|\boldsymbol{F}_{3}(\boldsymbol{x})\right\|_{L^{2}\left(E^{3}\right)}^{2} \leq 9\left\|\hat{K}_{\mathrm{s}}\right\|\left\|\boldsymbol{F}_{2}(\boldsymbol{x})\right\|_{L^{2}\left(E^{3}\right)}^{2},
$$


whence $\left\|\boldsymbol{F}_{3}(\boldsymbol{x})\right\| \rightarrow 0$ as $\alpha, \beta \rightarrow \infty$ owing to (4.22).

The remaining task is to show that $\left\|\boldsymbol{F}_{4}(\boldsymbol{x})\right\|$ vanishes in this limit. We partition $\boldsymbol{E}^{\mathrm{s}}(\boldsymbol{x} ; \alpha, \beta)$ into two parts as

$$
\boldsymbol{E}^{\mathrm{s}}(\boldsymbol{x} ; \alpha, \beta)=\boldsymbol{E}_{1}^{\mathrm{s}}(\boldsymbol{x} ; \alpha, \beta)+\boldsymbol{E}_{2}^{\mathrm{s}}(\boldsymbol{x} ; \alpha, \beta),
$$

where

$$
\boldsymbol{E}_{1}^{\mathrm{s}}(\boldsymbol{x} ; \alpha, \beta)=\mathbf{0} \text { for } \boldsymbol{x} \in E^{3} \backslash \Omega_{\delta} \text { and } \boldsymbol{E}_{2}^{\mathrm{s}}(\boldsymbol{x} ; \alpha, \beta)=\mathbf{0} \text { for } \boldsymbol{x} \in \Omega_{\delta} .
$$

Correspondingly, (4.10) is partitioned into

$$
\boldsymbol{F}_{4}(\boldsymbol{x})=\boldsymbol{F}_{41}(\boldsymbol{x})+\boldsymbol{F}_{42}(\boldsymbol{x}),
$$

where

$$
\boldsymbol{F}_{4 m}(\boldsymbol{x})=-\int_{Q} \frac{\hat{K}_{0}(\boldsymbol{x}-\boldsymbol{y})}{R^{2}}\left[\left(\hat{\varepsilon}^{\lambda}(\boldsymbol{y})-\hat{I}\right) \boldsymbol{E}_{m}^{\mathrm{s}}(\boldsymbol{y})\right] \mathrm{d} \boldsymbol{y} . \quad(m=1,2)
$$

The estimation of the norm of $\boldsymbol{F}_{4}(\boldsymbol{x})$ follows from separate estimation of each term since $\left\|\boldsymbol{F}_{4}(\boldsymbol{x})\right\|_{L^{2}(Q)}^{2} \leq\left\|\boldsymbol{F}_{41}(\boldsymbol{x})\right\|_{L^{2}(Q)}^{2}+\left\|\boldsymbol{F}_{42}(\boldsymbol{x})\right\|_{L^{2}(Q)}^{2}$. Let $\left\|\hat{K}_{0 \mathrm{c}}\right\|$ be the norm of the kernel $\hat{K}_{0}(\boldsymbol{x}-\boldsymbol{y}) / R^{2}$ of the compact operator. The latter of (4.26) is bounded above as

$$
\begin{aligned}
\left\|\boldsymbol{F}_{42}(\boldsymbol{x})\right\|_{L^{2}(Q)} & \leq\left\|\hat{K}_{0 \mathrm{c}}\right\|_{L^{2}(Q)} \max _{\boldsymbol{x} \in Q}\left\|\hat{\varepsilon}^{\lambda}(\boldsymbol{x})-\hat{I}\right\|\left\|\boldsymbol{E}_{2}^{\mathrm{s}}(\boldsymbol{x})\right\|_{L^{2}(Q)}^{2} \\
& \leq\left\|\hat{K}_{0 \mathrm{c}}\right\|_{L^{2}(Q)} \max _{\boldsymbol{x} \in Q}\left\|\hat{\varepsilon}^{\lambda}(\boldsymbol{x})-\hat{I}\right\| \varepsilon(\delta, \beta) \rightarrow 0 \quad \text { as } \delta \rightarrow 0
\end{aligned}
$$

being the allowable limit as $\alpha, \beta \rightarrow \infty$.

For small values of $\delta, \Omega_{\delta} \subset \Omega_{\sqrt{\delta}}$, and according (4.25), $\boldsymbol{E}_{1}^{\mathrm{s}}(\boldsymbol{x} ; \alpha, \beta)=\mathbf{0}$ for $\boldsymbol{x} \in \Omega_{\sqrt{\delta}} \backslash \Omega_{\delta}$. We use this property to pose an upper bound on $\boldsymbol{F}_{41}(\boldsymbol{x})$. Introducing (4.24) into (4.27), we have

$$
\begin{aligned}
\left\|\boldsymbol{F}_{41}(\boldsymbol{x})\right\|_{L^{2}(Q)}^{2} & =\int_{Q}\left|\int_{\Omega_{\sqrt{\delta}}} \frac{\hat{K}_{0}(\boldsymbol{x}-\boldsymbol{y})}{R^{2}}\left[\left(\hat{\varepsilon}^{\lambda}(\mathbf{0})-\hat{I}\right) \boldsymbol{E}_{1}^{\mathrm{s}}(\boldsymbol{y})\right] \mathrm{d} \boldsymbol{y}\right|^{2} \mathrm{~d} \boldsymbol{x} \\
= & \int_{\Omega_{\sqrt{\delta}}}\left|\int_{\Omega_{\sqrt{\delta}}} \frac{\hat{K}_{0}(\boldsymbol{x}-\boldsymbol{y})}{R^{2}}\left[\left(\hat{\varepsilon}^{\lambda}(\mathbf{0})-\hat{I}\right) \boldsymbol{E}_{1}^{\mathrm{s}}(\boldsymbol{y})\right] \mathrm{d} \boldsymbol{y}\right|^{2} \mathrm{~d} \boldsymbol{x} \\
& +\int_{Q \backslash \Omega_{\sqrt{\delta}}}\left|\int_{\Omega_{\delta}} \frac{\hat{K}_{0}(\boldsymbol{x}-\boldsymbol{y})}{R^{2}}\left[\left(\hat{\varepsilon}^{\lambda}(\mathbf{0})-\hat{I}\right) \boldsymbol{E}_{1}^{\mathrm{s}}(\boldsymbol{y})\right] \mathrm{d} \boldsymbol{y}\right|^{2} \mathrm{~d} \boldsymbol{x}
\end{aligned}
$$

The first term is bounded from above by

$$
\left(\left\|\hat{K}_{0 \mathrm{c}}\right\|_{L^{2}\left(\Omega_{\sqrt{\delta}}\right)} \max _{\boldsymbol{x} \in Q}\left\|\hat{\varepsilon}^{\lambda}(\boldsymbol{x})-\hat{I}\right\|\left\|\boldsymbol{E}_{1}^{\mathrm{s}}(\boldsymbol{x})\right\|\right)^{2} .
$$

Since $\hat{K}_{0} / R^{2}$ is a compact operator, $\left\|\hat{K}_{0 \mathrm{c}}\right\|_{L^{2}\left(\Omega_{\sqrt{\delta}}\right)} \rightarrow 0$ as $\delta \rightarrow 0$, and as a consequence the first integral in (4.29) tends to zero in this limit. 
For the estimation of the last integral in (4.29), we invoke the inequality

$$
\frac{1}{R}=\frac{1}{|\boldsymbol{x}-\boldsymbol{y}|} \leq \frac{1}{\sqrt{\delta}-\delta} \quad \text { for } \boldsymbol{x} \in Q \backslash \Omega_{\sqrt{\delta}}, \boldsymbol{y} \in \Omega_{\delta} .
$$

With this help, we are lead for any point $\boldsymbol{x} \in Q \backslash \Omega_{\sqrt{\delta}}$ to

$$
\begin{gathered}
\left|\int_{\Omega_{\delta}} \frac{\hat{K}_{0}(\boldsymbol{x}-\boldsymbol{y})}{R^{2}}\left[\left(\hat{\varepsilon}^{\lambda}(\mathbf{0})-\hat{I}\right) \boldsymbol{E}_{1}^{\mathrm{s}}(\boldsymbol{y})\right] \mathrm{d} \boldsymbol{y}\right|^{2} \leq C_{1}^{2}\left|\int_{\Omega_{\delta}} \frac{1}{R^{2}}\right| \boldsymbol{E}_{1}^{\mathrm{s}}(\boldsymbol{y})|\mathrm{d} \boldsymbol{y}|^{2} \\
\leq C_{1}^{2} \int_{\Omega_{\delta}} \frac{1}{R^{4}} \mathrm{~d} \boldsymbol{y} \int_{\Omega_{\delta}}\left|\boldsymbol{E}_{1}^{\mathrm{s}}(\boldsymbol{y})\right|^{2} \mathrm{~d} \boldsymbol{y} \leq \frac{C_{1}^{2}}{\delta^{2}(1-\sqrt{\delta})^{4}} \frac{4 \pi \delta^{3}}{3}\left\|\boldsymbol{E}^{\mathrm{S}}(\boldsymbol{x})\right\|^{2} .
\end{gathered}
$$

which tends to 0 in the limit of $\delta \rightarrow 0$. Here

$$
C_{1}=\max _{\boldsymbol{x}, \boldsymbol{y} \in Q}\left\|\hat{K}_{0}(\boldsymbol{x}-\boldsymbol{y})\right\| \max _{\boldsymbol{x} \in Q}\left\|\hat{\varepsilon}^{\lambda}(\boldsymbol{x})-\hat{I}\right\|,
$$

with $\left\|\hat{K}_{0}(\boldsymbol{x})\right\|$ being the norm of the tensor $\hat{K}_{0}(\boldsymbol{x})$, is a finite constant.

In this way, we have completed the proof of (4.4) in the constrained limit (4.1). Remembering that the operator $\hat{A}^{\lambda}$ is coined by replacing $\hat{\varepsilon}_{\mathrm{r}}$ by $\hat{\varepsilon}^{\lambda}$ in $(2.9)$, a symbolic representation of (2.5), it follows from (4.4) that

$$
\lim _{n \rightarrow \infty}\left\|\hat{A} \boldsymbol{E}^{\mathrm{S}}\left(\boldsymbol{x} ; \alpha_{n}, \beta_{n}\right)-\lambda \boldsymbol{E}^{\mathrm{S}}\left(\boldsymbol{x} ; \alpha_{n}, \beta_{n}\right)\right\|_{L^{2}(Q)}=0,
$$

in the same limit. For a point (3.2) of the essential spectrum for the anisotropic medium realizable with a normalised wavenumber $\boldsymbol{q}^{0} \in S^{2}$ and with monotonically increasing parameters $\left\{\alpha_{n}\right\}$ and $\left\{\beta_{n}\right\}$ given by (4.1), the corresponding singular mode is provided by the limit $n \rightarrow \infty$ of the sequence $\left\{\boldsymbol{E}^{\mathrm{s}}\left(\boldsymbol{x} ; \alpha_{n}, \beta_{n}\right)\right\}$ defined by formulae (3.11) and (3.12). We repeat that this sequence does not have a convergent subsequence in the Hilbert space.

\section{Singular modes in plasma}

From the physical viewpoint, worth pursuing is the possibility of $\lambda=0$ being the essential spectrum (3.2). If this happens to be the case, the singular mode possibly manifests itself in a certain medium. Actually, (2.9) reads $\hat{A} \boldsymbol{E}^{\mathrm{s}}=\mathbf{0}$ in such a case and the singular mode $\boldsymbol{E}^{\mathrm{s}}$ is realizable as an electric field confined at the singular point in $Q$ without having to send the external electric field $\boldsymbol{E}^{0}$.

We illustrate this possibility with a three-dimensional anisotropic medium of plasma. In accordance with the singular modes treated in this paper, it may be assumed, without loss of generality, that the external magnetic field is unidirectional along say the $z$ axis in Cartesian coordinates, that is, $\boldsymbol{H}^{0}=H^{0} \boldsymbol{e}_{3}$ where $\boldsymbol{e}_{3}$ is the unit vector in the $z$ direction. We pick out the following form of the Cartesian components of the dielectric permittivity tensor $\hat{\varepsilon}_{\mathrm{r}}$, normalised by $\varepsilon_{0}$, at the point $\boldsymbol{x}=\mathbf{0}$ (Ginzburg 1962):

$$
\begin{array}{ll}
\varepsilon_{11}=\varepsilon_{22}=1-\frac{\omega_{\mathrm{p}}^{2}(\omega-\mathrm{i} \nu)}{\omega\left[(\omega-\mathrm{i} \nu)^{2}-\omega_{H}^{2}\right]}, & \varepsilon_{33}=1-\frac{\omega_{\mathrm{p}}^{2}}{\omega(\omega-\mathrm{i} \nu)}, \\
\varepsilon_{12}=-\varepsilon_{21}=-\mathrm{i} \frac{\omega_{\mathrm{p}} \omega_{H}}{\omega\left[(\omega-\mathrm{i} \nu)^{2}-\omega_{H}^{2}\right]}, & \varepsilon_{13}=\varepsilon_{31}=\varepsilon_{23}=\varepsilon_{32}=0 .
\end{array}
$$


Here $\omega_{\mathrm{p}}$ is the plasma frequency, $\omega_{H}$ is the Lamor frequency, or the rotation frequency of the electrons around the external magnetic field, and $\nu$ is the collision frequency of the electrons to the ions. The first two are defined by

$$
\omega_{\mathrm{p}}^{2}=\frac{4 \pi e^{2} N}{m}, \quad \omega_{H}=\frac{\left|e H_{0}\right|}{m c},
$$

where $e$ and $m$ are the charge and the mass of the electron, respectively, and $N$ is the average number, per unit volume, of the electrons in the plasma.

According to (3.2), we seek the set of the normalised wavenumber $\boldsymbol{q}$ satisfying

$$
\boldsymbol{q} \cdot \hat{\varepsilon}_{\mathrm{r}} \boldsymbol{q}=\sum_{m=1}^{3} \sum_{n=1}^{3} \varepsilon_{m n}(\boldsymbol{x}) q_{m} q_{n}=0 .
$$

For the antisymmetric tensor (5.1), the off-diagonal components $\varepsilon_{12}$ and $\varepsilon_{21}$ do not contribute to the spectrum, leaving $\boldsymbol{q} \cdot \hat{\varepsilon}_{\mathrm{r}} \boldsymbol{q}=\varepsilon_{11} q_{1}^{2}+\varepsilon_{22} q_{2}^{2}+\varepsilon_{33} q_{3}^{2}$. After some algebra, we obtain

$$
\begin{aligned}
& \operatorname{Re}\left[\boldsymbol{q} \cdot \hat{\varepsilon}_{\mathrm{r}} \boldsymbol{q}\right]=\left[1-\frac{\omega_{\mathrm{p}}^{2}\left(\omega^{2}+\nu^{2}-\omega_{H}^{2}\right)}{\left(\omega^{2}-\nu^{2}-\omega_{H}^{2}\right)^{2}+4 \omega^{2} \nu^{2}}\right]\left(q_{1}^{2}+q_{2}^{2}\right)+\left(1-\frac{\omega_{\mathrm{p}}^{2}}{\omega^{2}+\nu^{2}}\right) q_{3}^{2}, \\
& \operatorname{Im}\left[\boldsymbol{q} \cdot \hat{\varepsilon}_{\mathrm{r}} \boldsymbol{q}\right]=-\frac{\nu \omega_{\mathrm{p}}^{2}\left(\omega^{2}+\nu^{2}+\omega_{H}^{2}\right)}{\omega\left(\omega^{2}-\nu^{2}-\omega_{H}^{2}\right)^{2}+4 \omega^{3} \nu^{2}}\left(q_{1}^{2}+q_{2}^{2}\right)-\frac{\nu \omega_{\mathrm{p}}^{2}}{\omega\left(\omega^{2}+\nu^{2}\right)} q_{3}^{2}
\end{aligned}
$$

It is evident from (5.4) that the presence of the energy loss $(\nu>0)$ prohibits emergence of the singular mode. Accordingly, we restrict ourselves to a plasma medium free from the loss $(\nu=0)$. In such a case, $(5.4)$ is reduced to

$$
\boldsymbol{q} \cdot \hat{\varepsilon}_{\mathrm{r}} \boldsymbol{q}=\left(1-\frac{\omega_{\mathrm{p}}^{2}}{\omega^{2}-\omega_{H}^{2}}\right)\left(q_{1}^{2}+q_{2}^{2}\right)+\left(1-\frac{\omega_{\mathrm{p}}^{2}}{\omega^{2}}\right) q_{3}^{2} .
$$

By adjusting the disposable parameters $\omega$ and $\boldsymbol{q}$, subject to $|\boldsymbol{q}|=1$, the singular modes with $\lambda=0$ are realizable in this anisotropic plasma. Suppose further that $\omega_{\mathrm{p}} \neq 0, \omega_{H} \neq 0$. For the lossless medium, the imaginary part of (5.4) does not give any condition, and $\lambda=0$ occurs generically on the circles $q_{1}^{2}+q_{2}^{2}=1-q_{3}^{2}$, lying on the unit sphere $S^{2}$, with $q_{3}$ provided by

$$
q_{3}= \pm \frac{|\omega|}{\omega_{\mathrm{p}} \omega_{H}} \sqrt{\omega_{\mathrm{p}}^{2}+\omega_{H}^{2}-\omega^{2}}
$$

The reality of $q_{3}$ requires $\omega^{2} \leq \omega_{\mathrm{p}}^{2}+\omega_{H}^{2}$, and the restriction $\left|q_{3}\right| \leq 1$ requires $\left(\omega^{2}-\omega_{\mathrm{p}}^{2}\right)\left(\omega^{2}-\omega_{H}^{2}\right) \geq 0$. Thus we reach a conclusion that the singular mode with $\lambda=0$ is realizable for the anisotropic medium of plasma characterized by (5.1) in either of the frequency ranges

$$
\begin{gathered}
|\omega| \leq \min \left\{\omega_{\mathrm{p}}, \omega_{H}\right\}, \\
|\omega| \geq \max \left\{\omega_{\mathrm{p}}, \omega_{H}\right\} \text { but with } \omega^{2} \leq \omega_{\mathrm{p}}^{2}+\omega_{H}^{2} .
\end{gathered}
$$

In the first range (5.7), (5.3) expresses two circles with $q_{3}$ given by (5.6) and therefore with radius given by $\sqrt{\left(\omega_{\mathrm{p}}^{2}-\omega^{2}\right)\left(\omega_{H}^{2}-\omega^{2}\right)} /\left(\omega_{\mathrm{p}} \omega_{H}\right)$. When the equality holds 


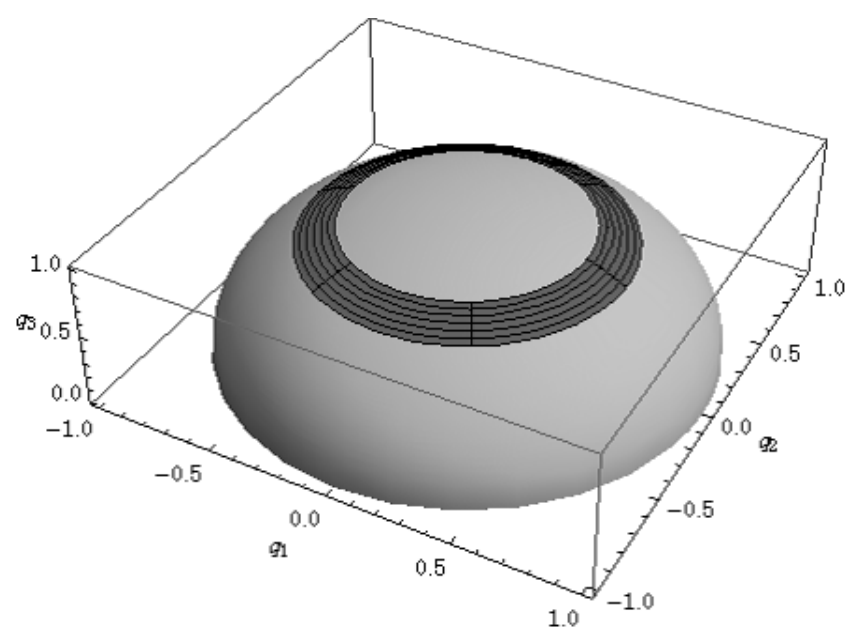

Figure 1. Perspective view of the set $U_{\boldsymbol{q}^{0}}(\alpha)$ (the dark region), located on the northern hemisphere. We take $\alpha=2$.

in (5.7), $q_{3}= \pm 1$ and the two circles shrink to two points $\boldsymbol{q}= \pm(0,0,1)$. In the other extreme, $\omega=0$, (5.3) expresses the equator circle $q_{3}=0$ and $q_{1}^{2}+q_{2}^{2}=1$. In the second range $(5.8),(5.3)$ expresses two circles of radius $\sqrt{\left(\omega^{2}-\omega_{\mathrm{p}}^{2}\right)\left(\omega^{2}-\omega_{H}^{2}\right)} /\left(\omega_{\mathrm{p}} \omega_{H}\right)$. At the upper limit of the frequency $\left(\omega^{2}=\omega_{\mathrm{p}}^{2}+\omega_{H}^{2}\right)$, it reduces to the equator circle $q_{3}=0$ and $q_{1}^{2}+q_{2}^{2}=1$.

To have an idea, we depict the region $U_{\boldsymbol{q}^{0}}(\alpha)$ defined by $(3.9)$, with $\varepsilon_{l m}^{\lambda}(\mathbf{0})$ replaced by $\hat{\varepsilon}_{\mathrm{r}}(\mathbf{0})$, for the singular mode attained in the range (5.7). We may take $\omega_{H}=\omega_{\mathrm{p}}$ and $\omega=\omega_{\mathrm{p}} / \sqrt{2}$. With this choice, (5.5) reads

$$
\boldsymbol{q} \cdot \hat{\varepsilon}_{\mathrm{r}} \boldsymbol{q}=3\left(q_{1}^{2}+q_{2}^{2}\right)-q_{3}^{2} .
$$

Taking $q_{1}^{2}+q_{2}^{2}+q_{3}^{2}=1$ into account, the essential spectrum $\lambda=0$ makes its appearance on the circles, lying on $S^{2}$, with $q_{3}= \pm \sqrt{3} / 2$. The condition $0 \leq$ $\boldsymbol{q} \cdot \hat{\varepsilon}_{\mathrm{r}} \boldsymbol{q} \leq 1 / \alpha$ leads to the zonal strip regions, for $U_{\boldsymbol{q}^{0}}(\alpha)$,

$$
\frac{\sqrt{3}}{2}\left(1-\frac{1}{3 \alpha}\right)^{1 / 2} \leq q_{3} \leq \frac{\sqrt{3}}{2}, \quad-\frac{\sqrt{3}}{2} \leq q_{3} \leq-\left(1-\frac{1}{3 \alpha}\right)^{1 / 2} .
$$

The area of the northern region $\left(q_{3}>0\right)$ in $U_{\boldsymbol{q}^{0}}(\alpha)$ is calculated to be

$$
S(\alpha)=\sqrt{3} \pi\left\{1-\left(1-\frac{1}{3 \alpha}\right)^{1 / 2}\right\} \text {. }
$$

For $\alpha \gg 1, S(\alpha) \approx \pi /(2 \sqrt{3} \alpha)$, being proportional to $\alpha^{-1}$ as expected. Figure 1 draws $U_{\boldsymbol{q}^{0}}(\alpha)$, as the dark zonal band, lying on the northern hemisphere $\left(q_{3}>0\right)$, by setting $\alpha=2$.

We close this section with a speculation of how the singular modes for $\lambda=0$ look like. The Maxwell equations are linear partial differential equations which admit a superposition of the electromagnetic waves without making any assumptions on their amplitudes. The frequency of the realizable modes is restricted to $\omega \in\left[\omega_{1}, \omega_{2}\right]$, 
where $\omega_{1}$ and $\omega_{2}$ are the lower and the upper bounds on the frequency determined by (5.7) or (5.8). Then by using the Fourier transform in time, we can construct the following singular electromagnetic field, at a fixed point $\boldsymbol{x}$ in $Q$,

$$
\boldsymbol{E}^{\mathrm{s}}(\boldsymbol{x}, t)=\int_{\omega_{1}}^{\omega_{2}} \boldsymbol{E}^{\mathrm{s}}(\boldsymbol{x}, \omega) \exp (-\mathrm{i} \omega t) \mathrm{d} \omega,
$$

where $\boldsymbol{E}^{\mathrm{s}}(\boldsymbol{x}, \omega)$ is the time-Fourier representation for the electric field of the singular mode given by the limit of (3.12). Appealing to an analogy with the uncertainty principle of the quantum mechanics, it is conceivable that, if the external field is switched off, these electromagnetic field would exist only in a limited time interval of order

$$
\frac{2 \pi}{\omega_{2}-\omega_{1}}
$$

\section{Summary and discussions}

We have explicitly constructed the singular mode or the singular eigenfunction corresponding to the continuous essential spectrum (1.1) of the singular volume integral operator $\hat{A}$, defined by the left hand side of (2.5), for the scattering of a electromagnetic monochromatic wave by a compact three-dimensional body with inhomogeneous and anisotropic dielectric permittivity, and have thus achieved an extension of the previous result for the isotropic case obtained by Budko \& Samokhin (2007). Our success hinges upon exploitation of the integral equation rather than of the Maxwell equations in their original form. Only when we work out the singular integral equation in the Fourier space, the explicit form of the essential spectrum (1.1) is at our hand for a general form of the permittivity tensor field which is Hölder continuous throughout the whole space. The framework of the integral equation renders it feasible to construct explicit form of the singular modes as well for isotropic dielectric scatterers (Budko \& Samokhin 2006b). This feasibility carries over to the anisotropic case as dealt with in this paper.

The salient feature of the singular mode is the localised, at a point, amplitude; the electric field of finite energy is confined to a point. To gain a insight into the formation of a pointwise localised wave, we give a crude argument. It is quite common that an essential continuous spectrum emerges at an accumulation point of discrete spectra (Budko \& Samokhin 2006a, 2006b). In other words, an essential spectrum is embedded in a dense set of discrete spectra. The essential spectrum becomes predominant at low frequencies. Suppose that $\lambda=0$ belongs to the essential spectrum. There are then an infinite number of discrete spectra $\left\{\lambda_{n} \mid n \in \mathbb{Z}\right\}$ of infinitesimal magnitude, that is, $\left|\lambda_{n}\right| \ll 1$ for all $n \in \mathbb{Z}$. Since the operator $\hat{A}$ defined by (2.9) is linear, in accordance with the linearity of the Maxwell equations, a superposition of the corresponding eigenfuction $\boldsymbol{E}_{n}(\boldsymbol{x})$ satisfies

$$
\hat{A} \sum_{n=-\infty}^{\infty} a_{n} \boldsymbol{E}_{n}(\boldsymbol{x})-\sum_{n=-\infty}^{\infty} \lambda_{n} a_{n} \boldsymbol{E}_{n}(\boldsymbol{x})=\mathbf{0}
$$

for arbitrary constants $\left\{a_{n}(\in \mathbb{C})\right\}$. In view of $\left|\lambda_{n}\right| \ll 1$ for all $n,(6.1)$ gives way to $\hat{A} \sum a_{n} \boldsymbol{E}_{n} \approx \mathbf{0}$, and hence we could think of $\sum a_{n} \boldsymbol{E}_{n}$ as an approximate eigenfunction associated with eigenvalue 0 . To be specific, we restrict ourselves to a 
one-dimensional periodic system, with period $2 \pi$, and take $\{\exp (\mathrm{i} n x) \mid n \in \mathbb{Z}\}$ eigenfunctions with their eigenvalues accumulating to $\lambda_{n} \rightarrow 0$ as $n \rightarrow \pm \infty$. If we set, for instance, $a_{n}=1 /(2 \pi)$ for all $n \in \mathbb{Z}$ with this setting, the eigenfunction becomes

$$
\frac{1}{2 \pi}\left(\cdots+\mathrm{e}^{-2 \mathrm{i} x}+\mathrm{e}^{-\mathrm{i} x}+1+\mathrm{e}^{\mathrm{i} x}+\mathrm{e}^{2 \mathrm{i} x}+\cdots\right)=\delta(x),
$$

being the Fourier series representation of the Dirac delta function for a periodic function with period $2 \pi$ in $x$. It should be born in mind that each element $\exp (\mathrm{i} n x)$ belongs to the Hilbert space but that the summation of an infinite number of elements is not necessarily so, depending on the choice of $\left\{a_{n}\right\}$. Although this argument may serve as a hint for a possible manner of how the localised mode is emerged, care should be excised as regards an important physical aspect. One of important feature of our singular mode is that the total electromagnetic energy is finite in spite of the localisation of the wave at a point, which is traced back to the manner of construction endowed with the condition (3.1) or (3.13). From this energetic viewpoint, our singular mode bears closer resemblance to the square root of the Dirac delta function as expounded by Budko \& Samokhin (2006a, 2007).

Singular modes represented explicitly in a tidy form are limited in number. Among them are the Van Kampen mode of the Vlasov equation for the plasma oscillations (Van Kampen 1955; Case 1959) and its generalization to Rayleigh's equation for inviscid waves on a parallel shear flow (Balmforth \& Morrison 1995; Hirota \& Fukumoto 2008a, 2008b). The singular mode presented in $\S 3$ should be added, along with the isotropic one obtained by Budko \& Samokhin (2007), to this list. The singular modes are indispensable to supply a complete set of eigenfunctions for describing evolution of an arbitrarily given initial wave. In general, fluids and plasmas have an abundance of continuous spectra, many of them being unavailable explicitly. To explore the stability of motion of fluids and plasmas, the continuous spectra and their singular modes are currently being under intensive investigation numerically as well as analytically.

Rapid advancement of the nano-technology makes possible to design and create exotic materials, like the photonic crystals, endowed with anomalous dielectric permittivity and magnetic permeability. We are thus requested to widen the knowledge of the electromagnetic-wave spectra. This paper has disclosed the singular mode in its naked form for the electromagnetic scattering problem. The role that this singular mode plays of in practical situations is yet to be explored. This and other aspects call for individual treatment.

Y. F. was supported in part by a Grant-in-Aid for Scientific Research from the Japan Society for the Promotion of Science (Grant No. 21540390). A. B. S. would also like to express his gratitude to the Japan Society for the Promotion of Science for the invitation and for the financial support for two-month stay at Kyushu University.

\section{References}

Balmforth, N. J. and Morrison, P. J. 1995 Normal modes and continuous spectra, Ann. New York Academy of Sciences 773, 80-94.

Berry, M. V. and Dennis, M. R. 2003 The optical singularities of birefringent dichroic chiral crystals. Proc. R. Soc. Lond. A 459, 1261-1292. 
Budko, N. V. and Samokhin, A. B. 2006a Classification of electromagnetic resonances in finite inhomogeneous three-dimensional structures. Phys. Rev. Lett. 96, 023904.

Budko, N. V. and Samokhin, A. B. 2006b Spectrum of the volume integral operator of electromagnetic scattering. SIAM J. Sci. Comput. 28, 682-700.

Budko, N. V. and Samokhin, A. B. 2007 Singular modes of the electromagnetic field. J. Phys. A: Math. Theor. 40, 6239-6250.

Case, K. M. 1959 Plasma oscillations. Ann. Phys. 7, 349-364.

Case, K. M. 1960 Stability of inviscid Couette flow. Phys. Fluids 3, 143-148.

Cubukcu, E., Aydin, K., Ekmel Ozbay, E., Foteinopoulou, S. and Soukoulis, C. M. 2003 Electromagnetic waves: negative refraction by photonic crystals. Nature 423, 604-605.

Ginzburg, V. L. 1962 Propagation of Electromagnetic Waves in Plasma, New York: Gordon and Breach Science Publishers Ltd.

Hirota, M. and Fukumoto, Y. 2008a Energy of hydrodynamic and magnetohydrodynamic waves with point and continuous spectra. J. Math. Phys. 49, 083101.

Hirota, M. and Fukumoto, Y. 2008b Action-angle variables for the continuous spectrum of ideal magnetohydrodynamics. Phys. Plasmas 15, 122101.

Hislop, P. D. and Sigal, I. M. 1996 Introduction to Spectral Theory: With Applications to Schrödinger Operators, New York: Springer.

Maslowe, S. A. 1986. Critical layers in shear flow, Annu. Rev. Fluid. Mech. 18, 405-432.

McIver, M., Linton, M., McIver, P., Zhang, J. and Porter, R. 2001 Embedded trapped modes for obstacles in two-dimensional waveguides. Q. J. Mech. Appl. Maths 54, 273293.

Mikhilin, S. G. 1965. Multidimensional Singular Integrals and Integral Equations, New York: Pergamon Press.

Mikhilin, S. G. and Prösdorf, S. 1986. Singular integral equations, New York: AkademieVerlag.

Rahola, J. 2000 On the eigenvalues of the volume integral operator of electromagnetic scattering. SIAM J. Sci. Comput. 21, 1740-1754.

Samokhin, A. B. 1990 Investigation of problems of the diffraction of electromagnetic waves in locally non-uniform media. U.S.S.R. Comput. Maths. Math. Phys. 30, 80-90.

Samokhin, A. B. 2001 Integral equations and iteration methods in electromagnetic scattering, Utrecht, The Netherlands: VSP.

Samokhin, A. B. 2009 Volume Integral Equation Method in Problems of Mathematical Physics, COE Lecture Note Vol. 24, Fukuoka, Japan: Kyushu University.

Sugimoto, N. and Imahori, H. 2006 Localized mode of sound in a waveguide with Helmholtz resonators. J. Fluid Mech. 546, 89-111.

Van Kampen, N. G. 1955 On the stationary waves in plasmas. Physica 21, 949-943.

Article submitted to Royal Society 


\section{List of MI Preprint Series, Kyushu University}

The Global COE Program

Math-for-Industry Education \& Research Hub

MI

MI2008-1 Takahiro ITO, Shuichi INOKUCHI \& Yoshihiro MIZOGUCHI

Abstract collision systems simulated by cellular automata

MI2008-2 Eiji ONODERA

The intial value problem for a third-order dispersive flow into compact almost

Hermitian manifolds

MI2008-3 Hiroaki KIDO

On isosceles sets in the 4-dimensional Euclidean space

MI2008-4 Hirofumi NOTSU

Numerical computations of cavity flow problems by a pressure stabilized characteristiccurve finite element scheme

MI2008-5 Yoshiyasu OZEKI

Torsion points of abelian varieties with values in nfinite extensions over a padic field

MI2008-6 Yoshiyuki TOMIYAMA

Lifting Galois representations over arbitrary number fields

MI2008-7 Takehiro HIROTSU \& Setsuo TANIGUCHI

The random walk model revisited

MI2008-8 Silvia GANDY, Masaaki KANNO, Hirokazu ANAI \& Kazuhiro YOKOYAMA Optimizing a particular real root of a polynomial by a special cylindrical algebraic decomposition

MI2008-9 Kazufumi KIMOTO, Sho MATSUMOTO \& Masato WAKAYAMA

Alpha-determinant cyclic modules and Jacobi polynomials 
MI2008-10 Sangyeol LEE \& Hiroki MASUDA

Jarque-Bera Normality Test for the Driving Lévy Process of a Discretely Observed Univariate SDE

MI2008-11 Hiroyuki CHIHARA \& Eiji ONODERA

A third order dispersive flow for closed curves into almost Hermitian manifolds

MI2008-12 Takehiko KINOSHITA, Kouji HASHIMOTO and Mitsuhiro T. NAKAO

On the $L^{2}$ a priori error estimates to the finite element solution of elliptic problems with singular adjoint operator

MI2008-13 Jacques FARAUT and Masato WAKAYAMA

Hermitian symmetric spaces of tube type and multivariate Meixner-Pollaczek polynomials

MI2008-14 Takashi NAKAMURA

Riemann zeta-values, Euler polynomials and the best constant of Sobolev inequality

MI2008-15 Takashi NAKAMURA

Some topics related to Hurwitz-Lerch zeta functions

MI2009-1 Yasuhide FUKUMOTO

Global time evolution of viscous vortex rings

MI2009-2 Hidetoshi MATSUI \& Sadanori KONISHI

Regularized functional regression modeling for functional response and predictors

MI2009-3 Hidetoshi MATSUI \& Sadanori KONISHI

Variable selection for functional regression model via the $L_{1}$ regularization

MI2009-4 Shuichi KAWANO \& Sadanori KONISHI

Nonlinear logistic discrimination via regularized Gaussian basis expansions

MI2009-5 Toshiro HIRANOUCHI \& Yuichiro TAGUCHII

Flat modules and Groebner bases over truncated discrete valuation rings 
MI2009-6 Kenji KAJIWARA \& Yasuhiro OHTA

Bilinearization and Casorati determinant solutions to non-autonomous $1+1$ dimensional discrete soliton equations

\section{MI2009-7 Yoshiyuki KAGEI}

Asymptotic behavior of solutions of the compressible Navier-Stokes equation around the plane Couette flow

MI2009-8 Shohei TATEISHI, Hidetoshi MATSUI \& Sadanori KONISHI

Nonlinear regression modeling via the lasso-type regularization

MI2009-9 Takeshi TAKAISHI \& Masato KIMURA

Phase field model for mode III crack growth in two dimensional elasticity

MI2009-10 Shingo SAITO

Generalisation of Mack's formula for claims reserving with arbitrary exponents for the variance assumption

MI2009-11 Kenji KAJIWARA, Masanobu KANEKO, Atsushi NOBE \& Teruhisa TSUDA Ultradiscretization of a solvable two-dimensional chaotic map associated with the Hesse cubic curve

\section{MI2009-12 Tetsu MASUDA}

Hypergeometric T -functions of the q-Painlevé system of type $E_{8}^{(1)}$

MI2009-13 Hidenao IWANE, Hitoshi YANAMI, Hirokazu ANAI \& Kazuhiro YOKOYAMA A Practical Implementation of a Symbolic-Numeric Cylindrical Algebraic Decomposition for Quantifier Elimination

MI2009-14 Yasunori MAEKAWA

On Gaussian decay estimates of solutions to some linear elliptic equations and its applications

MI2009-15 Yuya ISHIHARA \& Yoshiyuki KAGEI

Large time behavior of the semigroup on $L^{p}$ spaces associated with the linearized compressible Navier-Stokes equation in a cylindrical domain 
MI2009-16 Chikashi ARITA, Atsuo KUNIBA, Kazumitsu SAKAI \& Tsuyoshi SAWABE Spectrum in multi-species asymmetric simple exclusion process on a ring

MI2009-17 Masato WAKAYAMA \& Keitaro YAMAMOTO

Non-linear algebraic differential equations satisfied by certain family of elliptic functions

MI2009-18 Me Me NAING \& Yasuhide FUKUMOTO

Local Instability of an Elliptical Flow Subjected to a Coriolis Force

MI2009-19 Mitsunori KAYANO \& Sadanori KONISHI

Sparse functional principal component analysis via regularized basis expansions and its application

MI2009-20 Shuichi KAWANO \& Sadanori KONISHI

Semi-supervised logistic discrimination via regularized Gaussian basis expansions

MI2009-21 Hiroshi YOSHIDA, Yoshihiro MIWA \& Masanobu KANEKO

Elliptic curves and Fibonacci numbers arising from Lindenmayer system with symbolic computations

MI2009-22 Eiji ONODERA

A remark on the global existence of a third order dispersive flow into locally Hermitian symmetric spaces

MI2009-23 Stjepan LUGOMER \& Yasuhide FUKUMOTO

Generation of ribbons, helicoids and complex scherk surface in laser-matter Interactions

MI2009-24 Yu KAWAKAMI

Recent progress in value distribution of the hyperbolic Gauss map

MI2009-25 Takehiko KINOSHITA \& Mitsuhiro T. NAKAO

On very accurate enclosure of the optimal constant in the a priori error estimates for $H_{0}^{2}$-projection 
MI2009-26 Manabu YOSHIDA

Ramification of local fields and Fontaine's property (Pm)

MI2009-27 Yu KAWAKAMI

Value distribution of the hyperbolic Gauss maps for flat fronts in hyperbolic three-space

MI2009-28 Masahisa TABATA

Numerical simulation of fluid movement in an hourglass by an energy-stable finite element scheme

MI2009-29 Yoshiyuki KAGEI \& Yasunori MAEKAWA Asymptotic behaviors of solutions to evolution equations in the presence of translation and scaling invariance

MI2009-30 Yoshiyuki KAGEI \& Yasunori MAEKAWA

On asymptotic behaviors of solutions to parabolic systems modelling chemotaxis

MI2009-31 Masato WAKAYAMA \& Yoshinori YAMASAKI

Hecke's zeros and higher depth determinants

MI2009-32 Olivier PIRONNEAU \& Masahisa TABATA

Stability and convergence of a Galerkin-characteristics finite element scheme of lumped mass type

MI2009-33 Chikashi ARITA

Queueing process with excluded-volume effect

MI2009-34 Kenji KAJIWARA, Nobutaka NAKAZONO \& Teruhisa TSUDA

Projective reduction of the discrete Painlevé system of type $\left(A_{2}+A_{1}\right)^{(1)}$

MI2009-35 Yosuke MIZUYAMA, Takamasa SHINDE, Masahisa TABATA \& Daisuke TAGAMI Finite element computation for scattering problems of micro-hologram using DtN map 
MI2009-36 Reiichiro KAWAI \& Hiroki MASUDA

Exact simulation of finite variation tempered stable Ornstein-Uhlenbeck processes

MI2009-37 Hiroki MASUDA

On statistical aspects in calibrating a geometric skewed stable asset price model

MI2010-1 Hiroki MASUDA

Approximate self-weighted LAD estimation of discretely observed ergodic OrnsteinUhlenbeck processes

MI2010-2 Reiichiro KAWAI \& Hiroki MASUDA

Infinite variation tempered stable Ornstein-Uhlenbeck processes with discrete observations

MI2010-3 Kei HIROSE, Shuichi KAWANO, Daisuke MIIKE \& Sadanori KONISHI Hyper-parameter selection in Bayesian structural equation models

MI2010-4 Nobuyuki IKEDA \& Setsuo TANIGUCHI

The Itô-Nisio theorem, quadratic Wiener functionals, and 1-solitons

MI2010-5 Shohei TATEISHI \& Sadanori KONISHI

Nonlinear regression modeling and detecting change point via the relevance vector machine

MI2010-6 Shuichi KAWANO, Toshihiro MISUMI \& Sadanori KONISHI

Semi-supervised logistic discrimination via graph-based regularization

MI2010-7 Teruhisa TSUDA

UC hierarchy and monodromy preserving deformation

MI2010-8 Takahiro ITO

Abstract collision systems on groups

MI2010-9 Hiroshi YOSHIDA, Kinji KIMURA, Naoki YOSHIDA, Junko TANAKA \& Yoshihiro MIWA

An algebraic approach to underdetermined experiments 
MI2010-10 Kei HIROSE \& Sadanori KONISHI

Variable selection via the grouped weighted lasso for factor analysis models

MI2010-11 Katsusuke NABESHIMA \& Hiroshi YOSHIDA

Derivation of specific conditions with Comprehensive Groebner Systems

MI2010-12 Yoshiyuki KAGEI, Yu NAGAFUCHI \& Takeshi SUDOU

Decay estimates on solutions of the linearized compressible Navier-Stokes equation around a Poiseuille type flow

MI2010-13 Reiichiro KAWAI \& Hiroki MASUDA

On simulation of tempered stable random variates

MI2010-14 Yoshiyasu OZEKI

Non-existence of certain Galois representations with a uniform tame inertia weight

MI2010-15 Me Me NAING \& Yasuhide FUKUMOTO

Local Instability of a Rotating Flow Driven by Precession of Arbitrary Frequency

MI2010-16 Yu KAWAKAMI \& Daisuke NAKAJO

The value distribution of the Gauss map of improper affine spheres

MI2010-17 Kazunori YASUTAKE

On the classification of rank 2 almost Fano bundles on projective space

MI2010-18 Toshimitsu TAKAESU

Scaling limits for the system of semi-relativistic particles coupled to a scalar bose field

MI2010-19 Reiichiro KAWAI \& Hiroki MASUDA

Local asymptotic normality for normal inverse Gaussian Lévy processes with high-frequency sampling

MI2010-20 Yasuhide FUKUMOTO, Makoto HIROTA \& Youichi MIE

Lagrangian approach to weakly nonlinear stability of an elliptical flow 
MI2010-21 Hiroki MASUDA

Approximate quadratic estimating function for discretely observed Lévy driven SDEs with application to a noise normality test

MI2010-22 Toshimitsu TAKAESU

A Generalized Scaling Limit and its Application to the Semi-Relativistic Particles System Coupled to a Bose Field with Removing Ultraviolet Cutoffs

MI2010-23 Takahiro ITO, Mitsuhiko FUJIO, Shuichi INOKUCHI \& Yoshihiro MIZOGUCHI Composition, union and division of cellular automata on groups

MI2010-24 Toshimitsu TAKAESU

A Hardy's Uncertainty Principle Lemma in Weak Commutation Relations of Heisenberg-Lie Algebra

MI2010-25 Toshimitsu TAKAESU

On the Essential Self-Adjointness of Anti-Commutative Operators

MI2010-26 Reiichiro KAWAI \& Hiroki MASUDA

On the local asymptotic behavior of the likelihood function for Meixner Lévy processes under high-frequency sampling

MI2010-27 Chikashi ARITA \& Daichi YANAGISAWA

Exclusive Queueing Process with Discrete Time

MI2010-28 Jun-ichi INOGUCHI, Kenji KAJIWARA, Nozomu MATSUURA \& Yasuhiro OHTA

Motion and Bäcklund transformations of discrete plane curves

MI2010-29 Takanori YASUDA, Masaya YASUDA, Takeshi SHIMOYAMA \& Jun KOGURE On the Number of the Pairing-friendly Curves

MI2010-30 Chikashi ARITA \& Kohei MOTEGI

Spin-spin correlation functions of the $q$-VBS state of an integer spin model

MI2010-31 Shohei TATEISHI \& Sadanori KONISHI

Nonlinear regression modeling and spike detection via Gaussian basis expansions 
MI2010-32 Nobutaka NAKAZONO

Hypergeometric $\tau$ functions of the $q$-Painlevé systems of type $\left(A_{2}+A_{1}\right)^{(1)}$

MI2010-33 Yoshiyuki KAGEI

Global existence of solutions to the compressible Navier-Stokes equation around parallel flows

MI2010-34 Nobushige KUROKAWA, Masato WAKAYAMA \& Yoshinori YAMASAKI

Milnor-Selberg zeta functions and zeta regularizations

MI2010-35 Kissani PERERA \& Yoshihiro MIZOGUCHI

Laplacian energy of directed graphs and minimizing maximum outdegree algorithms

MI2010-36 Takanori YASUDA

CAP representations of inner forms of $S p(4)$ with respect to Klingen parabolic subgroup

MI2010-37 Chikashi ARITA \& Andreas SCHADSCHNEIDER

Dynamical analysis of the exclusive queueing process

MI2011-1 Yasuhide Fukumoto \& Alexander B. Samokhin

Singular electromagnetic modes in an anisotropic medium 